\title{
Analysis of the Relationship Between the Science Research Laboratories and Science Education
}

\author{
Fu Wei, Pengguang Tang, Li Qian, Xie Hao, Fang Jian \\ Chongqing Internet of Things Engineering Technology Research Center
}

Key Lab of Network Control Tech.\&Ins., Chongqing University of P\&T, Chongqing, 400065, China

E-mail: 61677822@qq.com

Keyword: science research laboratory; science research science education; resource advantage

Abstract. This article focuses on the relationship between the science research and the science education in the science research laboratories, combining the resource advantages of the science research laboratory, depth research the importance of the science research laboratory in the science education.

\section{Introduction}

The discus about science and technology is the primary productivity has been widely recognized in the world. The status of science become more important and science also is the first push of human progress. The popularization of scientific knowledge is the only way to implement the push. As today's rapid development of hi-tech, the degree of science and technology popularization in a country determined its production and culture production level, as well as the nation's ability to create. Recently, with the continuous efforts from relative government department and amplitude workers, science popularization in China has been rapid development. Mainly in the follows:

Posses a relative perfect popular science system preliminary.

Government department strengthen the popular science work through establishing a certain legal system.

Posses a quantitative channel of information popular science.

Science popularization in China also has some problem compared with the developed countries of Europe and America. Science popularization in China mainly depends on the government investment, including Popular Science and Technology Museum or popular science base construction. The majority of the social forces in the science education of the investment ratio are too small. Our country has been calling the social power involved in popular science education actively. Although the main work of Science Research Laboratory is scientific research. It is also a social popular science power with rich research resources. There is a positive promoting effect about Science Education based on Research Laboratory's resources and advantages. It is not only good for scientific research itself, but also for our national popular science education work.

\section{Science Research Laboratory Is An Important Social Popular Science Power}

\section{A. Advantage of Science Research Laboratory}

Scientific research laboratory in China is mainly distributed in universities and research institutes, over each academic field. It is the cradle and enrichment land of new scientific and technological knowledge. Research Laboratory has the best experiments and teaching equipments, as well as the advantaged predominance of hardware and software. It will become a new form and resources for Science popularization. At the same time, Research Laboratory is a resource of domestic and international advanced scientific knowledge and information, with large technology professional. Researchers, as the scientific information and knowledge creators, will be accepted by public when them as popular science propagandists. Whether it is from the hardware or software resources, all of these can constitute a new social science popularization resource. It is an important basis of our popular science work [1]

For example, our lab is Key Laboratory of Network Control Tech.\&Ins., Chongqing University of P\&T. At the same time it is also Key Laboratory of Chongqing. We have undertaken many 
popular science projects actively from Chongqing Education Committee and Science Committee. Our team is composed with teachers and postgraduates, and we make full use of all scientific research resources of our laboratory to organize many large science popularization activities, open laboratory to public regularly. We display the Lab's science popularization resources and scientific research achievements by reports, exhibition plate and visit. It was generally praised by Chongqing citizens. It sufficient proofs the unique advantage of scientific research laboratory in the social science popularization work. It can promote the national popular science work.

B. Social Responsibility of Science Research Laboratory

With the growth of public demand for science technology and the starting of project how to improve citizen scientific quality, China have developed new popular science system by legislation and policy guidance, to arouse more social forces to participate in China's modernization of Science Popularization. Science Research Laboratory has frontier of technology knowledge and the high-end software and hardware research equipments have the decisive position in social force of China's many popular science power. Research lab is a very important social science resource, so the majority of research lab should be actively involved in our national science popularization and play a role to promote our country citizen science quality improvement.

\section{Mutual Promotion Between Research And Popular Science}

For a long time, the scientific researchers in China reached a misunderstanding. We pay more attention to science research than science popularization. The majority of Research Laboratory doesn't have a correct understanding of mutual promotion between Research and Popular Science. Technology innovation is the driving force of economic development, and Science Popularization is the booster of economic development. With the emergency of knowledge economy and the prompt development of technology and science, the step of knowledge innovation accelerate continuously. Talent and technology has become the most important foundation of the modern economic growth. According to statistics, Britain has $40 \%$ of the world's scientific achievements in the years 1660 -1730. So it was the most powerful nation. The United States of America in 1950 with 57\% of the world's scientific achievements, this is why it can be the leading economic power of world up to now. From the analysis of this aspect, the following tendencies are very obvious in the environment that developing economic and enhancing the comprehensive national strength. First is putting attention from the real wealth increment value to the potential incremental wealth: science and technology [2]. Second is from physical capital and other tangible wealth to technology, talent and other intangible wealth. Third is from equipment import to management technology software import. If from the cognitive perspective, technology innovation is the key of national comprehensive competitiveness. Science Popularization is a operation to make science and technology polarization, application and generalization. Any item of science and technology can't be mastered by people into the real productivity without popular science. The purpose of science and technology innovation is make science into productivity by popularization of science and technology [3]. Therefore, we should pay more attention to popular science work like a great importance to technological innovation. Otherwise economic development is the lack of stamina.

Research is continuously made new breakthroughs in science and technology. It is conducive to the promotion of the scientific knowledge and interest for public. Economy and science \& technology can't progress without advanced technology and process. We can' t live without research and science popularization is an important basis of science and technology innovation. The popularization of science and technology is a necessary link in the transformation of scientific and technology from the first productivity into real productivity. Without science popularization, there can be no sustained technological innovation.

We must not only attach importance to scientific and technological innovation, but also the science popularization. To enhance the strength of science and technology, we must raise the level of scientific research and improve the popular science level at the same time. Science is inseparable from the popular. Now scientific left the extensive participation and support of people will not called innovative research. China and the United States, Japan and other countries of the technology 
gap is not only reflected in the level of scientific research, as well as in popular science level. Only in the solid foundation which is based on science, there will be a great master of scientist and have innovation works.

\section{Conclusion}

Research laboratory as the main force of China's scientific and technological innovation has an inescapable responsibility of science popularization. Many researchers and scientists in Scientific Research Laboratory should give full play to the world of science and technology resources and talent advantages. They should pay more attention to substance, form and other aspect, do more work for knowledge dissemination and public understanding of science and technology. They play a positive guiding role in popular science work by show the scientific research achievements [4]. The majority of research laboratories should be combined with self-conditions, formed a set of independence stand on one's own science organization system to ensure sustainable scientific research personnel in the popular science work from the system. Key Lab of Network Control Tech.\&Ins., Chongqing University of P\&T actively participate in the Chongqing Municipal Science \&Technology Culture Festival and Eagle plan for cultivate students' innovative. Researchers in the lab make it a necessary work. All the popular science work is under the guidance of the teachers, and passed down by students. This makes us laboratory formed a benign circulation system of popular science. It is more conducive to participate in scientific research and popular science laboratory.

In a word, our research laboratory staff should have a correct understanding —-Innovation of science and technology to provide source and means for the popularization of science and technology; the popularization of science and technology to provide the power for the development of innovation of science and technology; both of them to promote the development of science and technology and social progress. Research and science popularization of Science supplement each other and parallel development. It is the effective way to unify capability of independent innovation and creativity of the whole society organically [5].

\section{Acknowledgment}

Popular science series of Internet of things (cstc2012gg-kplB40006), Scientific and technological research project (popular science class) of Chongqing.

Internet of things the interactive experience on the research and development of library science exhibits and teaching aids (cstc2012gg-kplB40005), scientific and technological research project (popular science class) of Chongqing.

IT popular science bases of Chongqing, key science base construction project of Chongqing.

Construction of automation graduate innovation education bases, key project of graduate education innovation of Chongqing University of P\&T.

Research and practice of the engineering training center construction (1201019), Chongqing higher education teaching reform research project.

\section{References}

[1] Liu Yanjun, Wu Chensheng, Wu Qiong, Zhang Luji, "Present situation, problems and Countermeasures of China's scientific research institutions to carry out the popular science work"[J] Association for science and Technology Forum (first half) 2010.02(07)

[2] Dai Wenying, Play to advantages to carry out Scientific Research Institute of education science activities [J] Basic education research 2002.05

[3] Hu Xiangming, China Heilongjiang 1997 total solar eclipse and comet activities [J] Acad2009.04 
[4] Ji Shiqiang, Ren Jianxia, Analysis of characteristics of modern science popularization [J] Journal of Ningbo radio and Television University 2006.03

[5] Qu Xiang, Zhang Zhi, Liu Biao, Liao Shan, Hong Tao, Carry forward the spirit of science, play the role of science popularization in Open Laboratory [J] Experimental technology and management 2010.06 